\title{
A Equação da Onda-imagem para Remigração na Profundidade em Meios Elipticamente Anisotrópicos
}

\author{
R. ALEIXO, J. SCHLEICHER ${ }^{2}$, Departamento de Matemática Aplicada, IMECC, \\ UNICAMP, 13083-970 Campinas, SP, Brasil.
}

\begin{abstract}
Resumo. A imagem de um refletor sísmico muda de posição na profundidade quando remigrada sob variação do modelo de velocidade. Esta "propagação" pode ser descrita por meio de uma equação diferencial parcial de segunda ordem semelhante à equação da onda acústica. Neste trabalho, levamos a dedução desta chamada equação da onda-imagem para meios elipticamente isotrópicos, utilizando as propriedades geométricas do problema da remigração sísmica. Como no caso isotrópico, a dedução utiliza uma metodologia que aplica a teoria dos raios padrão em ordem reverse. O objetivo é a construção de imagens do subsolo que correspondem a diferentes graus de anisotropia do meio, utilizando a propagação da imagem ao invés de múltiplas migrações.
\end{abstract}

\section{Introdução}

Neste trabalho levamos a dedução da equação da onda imagem para o problema de remigração na profundidade, anteriormente desenvolvida em meios isotrópicos [2, 4], para meios com anisotropia eliptica. O resultado é uma equação diferencial parcial de segunda ordem semelhante à equação da onda acústica. A dedução emprega, em ordem reversa, a metodologia desenvolvida na aplicação da teoria dos raios para a equação onda (veja, por exemplo, [1]).

O problema da remigração provém do processamento sísmico e o seu objetivo é a construção de uma nova imagem do subsolo a partir de uma imagem obtida anteriormente pelo processo de migração, utilizando outros parâmetros do meio [5]. Por migração se conhece, na geofísica, o processo que tem como objetivo a reconstrução de uma imagem das camadas geológicas no subsolo a partir da imagem distorcida no tempo, obtida mediante um levantamento sísmico, i.e., mediante geração de ondas no subsolo e registro do movimento resultante das partículas da superfície da terra. Para a realização da migração é necessário conhecer um modelo das velocidades de propagação das ondas no subsolo, as chamadas velocidades de migração.

Porém, o modelo de velocidade usado para a primeira migração, geralmente não é perfeito, resultando em uma imagem incorreta. Esta, por sua vez, pode fornecer informações que permitem a atualização do modelo de velocidade. Alternativamente,

\footnotetext{
1 aleixo@ime.unicamp.br

2 js@ime.unicamp.br
} 
é interessante possuir um leque de imagens referentes a diferentes modelos de velocidade, para escolher entre eles o geologicamente mais fidedigno ou aquele que melhor coincide com informações adicionais, tais como medidas em poços na área.

Desta forma, torna-se necessária a construção de uma nova imagem do subsolo referente a um modelo atualizado. Esta nova imagem pode ser construída mediante uma nova migração dos dados originais ou pelo processo de remigração $[2,4,5]$. Para esta atualização, foram sugeridos na literatura operadores diferenciais $[2,4]$ e integrais [5]. Jaya [6] estudou os primeiros e apresentou as primeiras aplicações práticas em dados reais. Baseado na equação diferencial de Fomel [2], Hubral et al. [4] observaram que as novas imagens de um refletor (i.e., uma fronteira entre camadas geológicas) para diferentes modelos de velocidade de migração comportam-se de maneira análoga à propagação de frentes de onda. Para entender melhor a analogia com a propagação de uma onda, veja a Figura 1. $\mathrm{Na}$ Figura 1(a) podemos ver, esquematicamente, como uma frente de onda se propaga. A mesma frente de onda é mos-

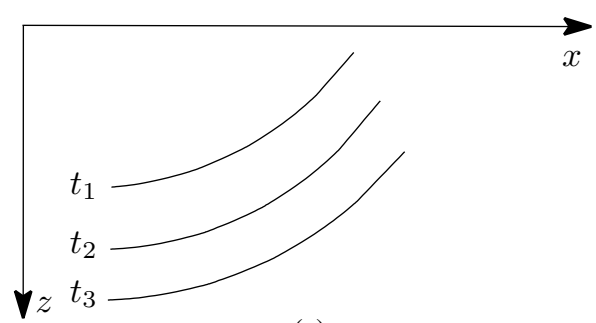

(a)

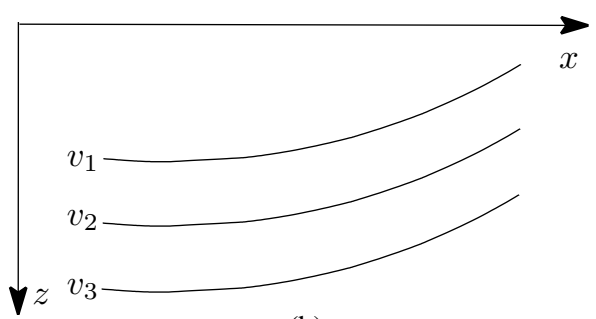

(b)

Figura 1: (a) Frentes de onda se propagando em três instantes de tempo diferentes. (b) Ondas-imagem em três velocidades de migração diferentes.

trada em três instantes de tempo diferentes. Por outro lado, vemos na Figura 1(b) três imagens migradas diferentes de um mesmo refletor sísmico, obtido com três modelos de velocidade de migração diferentes. Se compararmos a situação com a Figura 1(a), não é difícil aceitar que pode ser, conceitualmente, entendida como uma "onda se propagando". Neste caso é a imagem de um refletor sísmico que "propaga". Hubral et al. [4] deram a este fenômeno o nome de "onda-imagem". Da mesma forma que a Figura 1 (a) mostra três frentes de onda em três instantes do tempo, a Figura 1(b) mostra a frente da onda-imagem de três "instantes de velocidade de migração". A variável de propagação, que no caso da propagação de ondas físicas é representada pelo tempo, é, no caso das ondas-imagem, a velocidade de migração.

Os trabalhos citados acima estudam a propagação da onda-imagem em função do valor da velocidade de migração (considerada a mesma no modelo inteiro). Aqui estamos interessados na remigração em meios elipticamente anisotrópicos, sob variação do parâmetro de anisotropia. Definimos o parâmetro de elipticidade do meio como a razão entre os quadrados das velocidades na vertical e na horizontal. Chamamos este parâmetro de $\varphi$. Nosso objetivo é estudar a variação da imagem do refletor em função da variação deste parâmetro $\varphi$. Em outras palavras, $\varphi$ assume o papel da variável de propagação para a onda-imagem em meios elipticamente anisotrópicos. 


\section{Dedução da Onda-imagem}

Nesta seção descrevemos a propagação da onda-imagem como uma função da anisotropia do meio. Para tal, estudamos como um ponto isolado em uma imagem de um refletor se comporta quando a elipticidade do meio varia. Esta situação pode ser entendida de maneira análoga à propagação de uma onda de Huygens a partir de uma fonte secundária pontual. Esta descreve como um ponto isolado em uma frente de onda se comporta quando o tempo varia.

Deduzimos a equação da onda-imagem empregando a metodologia desenvolvida na aplicação da teoria dos raios para a equação onda [1]. Tal metodologia está resumida abaixo. A idéia é separar a onda em suas partes cinemática e dinâmica, i.e., tempo de trânsito e amplitude. Assim, a partir da equação onda, pode ser encontrada a equação iconal que descreve a cinemática da onda, isto é, a localização das frentes de onda. O procedimento se baseia na equação da onda acústica

$$
p_{x x}+p_{z z}=\frac{1}{v^{2}} p_{t t} .
$$

Uma solução aproximada da equação acima é obtida utilizando a candidata da teoria dos raios,

$$
p(x, z, t)=p_{0}(x, z) f[t-T(x, z)] .
$$

Aqui, $f(t)$ é um pulso de máxima freqüência supostamente fixo. As quantidades $p_{0}(x, z)$ e $T(x, z)$ representam o fator de amplitude e tempo de trânsito da onda. A candidata (2.2) é uma aproximação da solução da equação (2.1) em meios fracamente heterogêneos, onde o pulso pode mudar vagarosamente sua forma e amplitude ao longo de uma frente da onda $t=T(x, z)$. Sugerimos ao leitor interessado neste assunto procurar, por exemplo, o livro de Črvený [1].

Derivando a candidata (2.2) duas vezes em relação $x, z$ e $t$, e substituindo os resultados na equação $(2.1)$, temos

$p_{0}\left(T_{x}^{2}+T_{z}^{2}-v^{-2}\right) f^{\prime \prime}-\left[2\left(p_{0 x} T_{z}+p_{0 z} T_{z}\right)+p_{0}\left(T_{x x}+T_{z z}\right)\right] f^{\prime}+\left(p_{0 x x}+p_{0 z z}\right) f=0$.

Para que a equação (2.3) seja satisfeita para qualquer pulso $f$, cada coeficiente das derivadas de $f$ precisa zerar independentemente. A partir do coeficiente de $f^{\prime \prime}$ temos a equação iconal

$$
T_{x}^{2}+T_{z}^{2}=\frac{1}{v^{2}}
$$

A solução desta equação para a condição inicial de uma fonte pontual em $\left(x_{0}, z_{0}\right)$ no instante $t_{0}$ é a chamada onda de Huygens, $t=T\left(x, x_{0}, z_{0}\right)$. Ela representa a localização $z(x, t)$ da onda como o resultado de uma fonte pontual secundária em $\left(x_{0}, z_{0}\right)$. Tais fontes secundárias são excitadas a cada ponto de uma frente de onda se propagando. Para outras condições iniciais, a equação (2.4) descreve a cinemática de qualquer propagação de ondas.

Correspondentemente, a equação da onda-imagem em meios elipticamente anisotrópicos a ser determinada tem uma equação iconal associada. Esta descreve a cinemática da propagação onda-imagem, i.e., localiza as suas frentes de onda. Sua solução para uma fonte pontual pode ser chamada de onda-imagem de Huygens. 
Neste trabalho, ao contrário da ordem da abordagem acima, descrevemos primeiramente a onda de Huygens da onda-imagem. Posteriormente, obtemos a equação iconal por eliminação das constantes da condição inicial. Finalmente, estabelecemos a equação onda-imagem, sendo ela a equação diferencial parcial de segunda ordem mais simples com a propriedade de que a metodologia acima possa ser nela aplicada para reproduzir a equação iconal associada.

\subsection{Parametrização dos meios elipticamente anisotrópicos}

Aqui estamos interessados na remigração em meios elipticamente anisotrópicos. Estes são caracterizados por serem meios com simetria vertical. O seu tensor de elasticidade, normalizado na densidade, é dado por uma matriz da forma

$$
\mathbf{A}=\left(\begin{array}{cccccc}
A_{11} & A_{12} & A_{13} & 0 & 0 & 0 \\
A_{12} & A_{11} & A_{13} & 0 & 0 & 0 \\
A_{13} & A_{13} & A_{33} & 0 & 0 & 0 \\
0 & 0 & 0 & A_{44} & 0 & 0 \\
0 & 0 & 0 & 0 & A_{44} & 0 \\
0 & 0 & 0 & 0 & 0 & A_{66}
\end{array}\right)
$$

com as restrições

$$
\begin{aligned}
A_{12} & =A_{11}-2 A_{66}, \\
\left(A_{13}+A_{44}\right)^{2} & =\left(A_{11}-A_{44}\right)\left(A_{33}-A_{44}\right) .
\end{aligned}
$$

Desta forma, um meio com tal anisotropia é descrito por quatro parâmetros elásticos independentes.

Para fins de imageamento sísmico, o parâmetro do meio mais importante é a velocidade da propagação de ondas. Adicionamos aqui uma pequena uma discussão sobre a velocidade em meios com anisotropia elíptica. Mais detalhes sobre meios com anisotropia elíptica podem ser encontrados em [3] ou [7]. Nestes meios, o vetor velocidade de grupo da onda compressional ou quase-P (qP), $\vec{v}$, é dado por

$$
\vec{v}=\left(\frac{A_{11}}{V} \sin \phi, 0, \frac{A_{33}}{V} \cos \phi\right),
$$

onde $\phi$ é o ângulo entre a normal à frente de onda e o eixo $z, \mathrm{e}$

$$
V=\sqrt{A_{11} \sin ^{2} \phi+A_{33} \cos ^{2} \phi}
$$

é a velocidade de fase da onda.

Concluímos que o valor da velocidade de grupo varia com a direção de propagação de acordo com

$$
\|\vec{v}\|=v(\theta)=\frac{\sqrt{A_{11}^{2} \sin ^{2} \phi+A_{33}^{2} \cos ^{2} \phi}}{V}=\left[\frac{\sin ^{2} \theta}{A_{11}}+\frac{\cos ^{2} \theta}{A_{33}}\right]^{-1 / 2},
$$


onde $\theta$ é o ângulo entre o vetor da velocidade de grupo $\vec{v}$ e o eixo $z$, chamado de ângulo de propagação. A relação entre $\phi$ e $\theta$ é dada pela seguinte equação [7]

$$
\tan \theta=\frac{A_{11}}{A_{33}} \tan \phi .
$$

Em função da anisotropia, as velocidades de propagação de uma onda na vertical e na horizontal são diferentes. Pela equação (2.5) podemos ver que as velocidades nas direções vertical $(\theta=0)$ e horizontal $(\theta=\pi / 2)$ são

$$
v_{v}=\sqrt{A_{33}} \text { e } \quad v_{h}=\sqrt{A_{11}} .
$$

\subsection{Geometria do levantamento sísmico}

Analisamos a situação de um levantamento sísmico de afastamento nulo, onde o par coincidente de fonte e receptor está localizado na superfície da terra $(z=0)$ no ponto $S=(\xi, 0)$ (Figura 2).

Sendo $x$ e $z$ as coordenadas de um certo ponto $P$ no meio em questão e $\ell$ a sua distância da fonte $S$, tal que $\ell^{2}=(x-\xi)^{2}+z^{2}$, o ângulo de propagação de uma onda que propaga de $S=(\xi, 0)$ até $P=(x, z)$, satisfaz

$$
\cos \theta=\frac{z}{\ell} \quad \text { e } \quad \sin \theta=\frac{x-\xi}{\ell} .
$$

Para melhor visualização, veja a Figura 2.

Assim, obtemos a representação alternativa da velocidade de propagação, que depende do ângulo de propagação, em função das coorde-

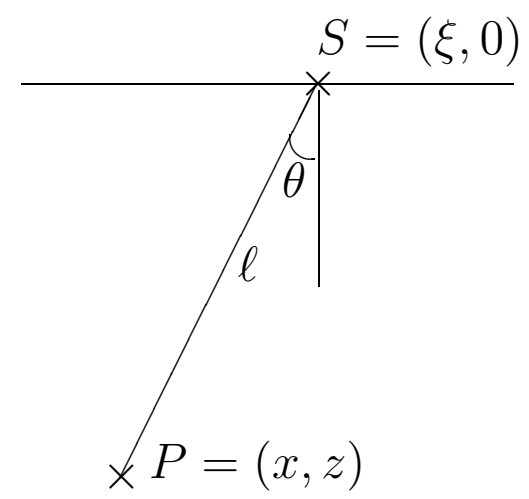

Figura 2: Representação gráfica do raio conectando a fonte $\mathrm{S}$ em $(\xi, 0)$ e o ponto $P=(x, z)$ no refletor sísmico. nadas do ponto $P$

$$
v(x, z)=\ell\left[\frac{(x-\xi)^{2}}{A_{11}}+\frac{z^{2}}{A_{33}}\right]^{-1 / 2}=\ell v_{v}\left[\varphi(x-\xi)^{2}+z^{2}\right]^{-1 / 2}
$$

onde introduzimos o parâmetro $\varphi=A_{33} / A_{11}=v_{v}^{2} / v_{h}^{2}$.

Com os resultados desta pequena discussão sobre a velocidade de propagação nesses meios em função da direção, podemos descrever o tempo de trânsito $T$ da onda emitida e registrada em $S=(\xi, 0)$ e refletida em $P$. A partir da fórmula para a velocidade, sabemos que o tempo que uma onda leva quando parte de uma fonte e chega em um receptor no mesmo ponto da fonte, é

$$
T(\xi ; x, z)=\frac{2 \ell}{v(x, z)}=\frac{2}{v_{v}}\left[\varphi(x-\xi)^{2}+z^{2}\right]^{1 / 2} .
$$

O fator 2 se deve à observação na equação $(2.5)$ que $v(\theta)=v(\theta+\pi)$, que implica que o tempo para a onda chegar ao ponto no refletor é o mesmo do ponto no refletor ao detector. 


\subsection{Remigração}

A remigração tenta estabelecer uma relação entre dois meios de propagação de ondas sísmicas, tais que os levantamentos sísmicos resultariam nos mesmos dados. Um destes meios representa o modelo de velocidade errado usado originalmente para a migração. O outro representa o modelo atualizado no qual a nova imagem do refletor precisa ser construída.

Supomos que a migração original tenha sido realizada com um modelo caracterizado pela mesma velocidade vertical $v_{v}$, mas uma outra elipticidade $\varphi_{0}$. Neste meio, o mesmo tempo $T$ da equação (2.7), consumido por uma outra onda refletida em outro ponto $P_{0}=\left(x_{0}, z_{0}\right)$ é dado por

$$
T\left(\xi ; x_{0}, z_{0}\right)=\frac{2 \ell_{0}}{v\left(x_{0}, z_{0}\right)}=\frac{2}{v_{v}}\left[\varphi_{0}\left(x_{0}-\xi\right)^{2}+z_{0}^{2}\right]^{1 / 2}
$$

A Figura 3(a) mostra o tempo descrito pela equação (2.8) para um conjunto realístico de parâmetros $\left(x_{0}, z_{0}, \varphi_{0}, v_{v}\right)$. Adotamos a convenção de chamar o meio com elipticidade $\varphi$ de meio $M$, e o meio com elipticidade $\varphi_{0}$ de meio $M_{0}$.

Onda-imagem de Huygens: Para deduzirmos a equação da onda-imagem, seguimos a metodologia proposta em [4]. Primeiramente, queremos achar todos o pontos $P=(x, z)$ no meio $M$ tais que o seu tempo de reflexão, descrito pela equação (2.7), seja igual ao tempo de reflexão (2.8) do ponto $P_{0}=$ $\left(x_{0}, z_{0}\right)$ no meio $M_{0}$. Em outras palavras, estamos interes-
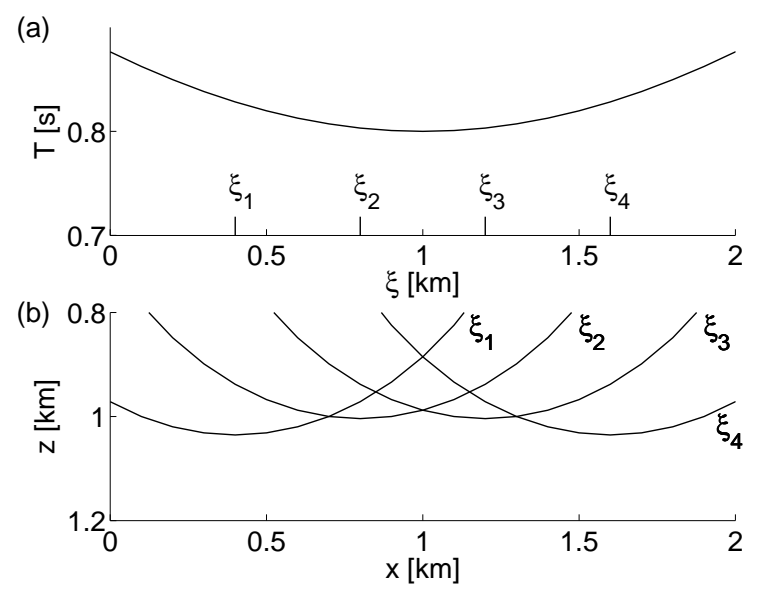

Figura 3: (a) Gráfico do tempo descrito pela equação (2.8) com $x_{0}=1 \mathrm{~km}, z_{0}=1 \mathrm{~km}, \varphi_{0}=$ $0.2, \varphi=0.8$ e $v_{v}=2.5 \mathrm{~km} / \mathrm{s}$. (b) Família de curvas dada pela equação $(2.9) \operatorname{com} x_{0}=1 \mathrm{~km}, z_{0}=$ $1 \mathrm{~km}, \varphi_{0}=0.2$ e $\varphi=0.8$ e o parâmetro $\xi$ assumindo os valores $0.4 \mathrm{~km}, 0.8 \mathrm{~km}, 1.2 \mathrm{~km}$ e $1.6 \mathrm{~km}$.

sados em localizar a chamada onda de Huygens para esta propagação da ondaimagem, i.e., o local da imagem $z(x)$ no "instante" $\varphi$, que se "originou" no "instante" $\varphi_{0}$ no ponto $P_{0}$. Para tal, igualamos os tempos $T$ das equações $(2.7)$ e (2.8), resultando em

$$
F(x, z, \xi, \varphi)=\varphi(x-\xi)^{2}+z^{2}-\varphi_{0}\left(x_{0}-\xi\right)^{2}-z_{0}^{2}=0 .
$$

Esta equação representa uma família de curvas $z(x ; \xi)$ que, para um $\xi$ fixo, conecta todos o pontos $P$ no meio $M$ que possuem o mesmo tempo de reflexão $T(\xi ; x, z)$ que $P_{0}$ no meio $M_{0}$. Na Figura $3(\mathrm{~b})$ vemos quatro curvas obtidas da equação (2.9) para diferentes valores de $\xi$. 
O conjunto de pontos $P$ tais que $T(\xi ; x, z)$ é igual a $T\left(\xi ; x_{0}, z_{0}\right)$ para todo $\xi$ é dado pelo envelope desta família de curvas $z(x ; \xi)$. Esta curva é a mencionada onda de Huygens da onda-imagem, uma vez que ela representa a imagem no meio $M$ do ponto $P_{0}$. Sabemos que a condição de envelope da família de curvas é

$$
\frac{\partial F}{\partial \xi}=0
$$

que, substituída na equação (2.9), fornece a curva desejada. Derivando a equação (2.9) com relação a $\xi$, obtemos

$$
-\varphi(x-\xi)+\varphi_{0}\left(x_{0}-\xi\right)=0 \quad \Rightarrow \quad\left(\varphi-\varphi_{0}\right) \xi=\varphi x-\varphi_{0} x_{0}
$$

Logo,

$$
\xi=\frac{\varphi x-\varphi_{0} x_{0}}{\left(\varphi-\varphi_{0}\right)}=\frac{\alpha x-x_{0}}{\alpha-1}=\frac{x_{0}-\alpha x}{1-\alpha},
$$

onde $\alpha=\varphi / \varphi_{0}$. De (2.9), concluímos que

$$
z^{2}=\varphi_{0}\left(x_{0}-\xi\right)^{2}+z_{0}^{2}-\varphi(x-\xi)^{2}
$$

e (2.10) fornece

$$
\begin{aligned}
\left(x_{0}-\xi\right)^{2} & =\left[x_{0}-\frac{x_{0}-\alpha x}{1-\alpha}\right]^{2}=\frac{\alpha^{2}}{(1-\alpha)^{2}}\left(x-x_{0}\right)^{2} \\
(x-\xi)^{2} & =\left[x-\frac{x_{0}-\alpha x}{1-\alpha}\right]^{2}=\frac{1}{(1-\alpha)^{2}}\left(x-x_{0}\right)^{2}
\end{aligned}
$$

A substituição destas relações em (2.11) resulta em

$$
\begin{aligned}
z^{2} & =z_{0}^{2}+\varphi_{0} \frac{\alpha^{2}}{(1-\alpha)^{2}}\left(x-x_{0}\right)^{2}-\varphi \frac{1}{(1-\alpha)^{2}}\left(x-x_{0}\right)^{2} \\
& =z_{0}^{2}-\frac{\varphi\left(x-x_{0}\right)^{2}}{1-\alpha}
\end{aligned}
$$

que pode ser reescrito como

$$
z=\sqrt{z_{0}^{2}+\varphi \varphi_{0} \frac{\left(x-x_{0}\right)^{2}}{\varphi-\varphi_{0}}}
$$

A equação acima descreve a localização da onda de Huygens da onda-imagem para a condição inicial $\left(x_{0}, z_{0} ; \varphi_{0}\right)$. Veja a Figura $4($ a) para vizualizar o formato do envelope das curvas dadas pela equação (2.9), ou seja, a onda-imagem de Huygens. A Figura 4(b) mostra vários destes envelopes para diferentes valores de $\varphi$. 
Equação iconal: Para deduzirmos a equação iconal, precisamos eliminar as constantes $x_{0}, z_{0}$ e $\varphi_{0}$ da equação (2.13) em favor de derivadas, para então podermos descrever a propagação da onda-imagem para qualquer condição inicial arbitrária. Para isso permitimos a $\varphi$ variar, ou seja, substituímos $\varphi=\Phi(x, z)$ na equação $(2.13)$, onde $\Phi(x, z)$ é o iconal da ondaimagem. Por diferenciação da equação resultante, encontramos uma equação diferencial para $\Phi$ cuja solução com as condições iniciais $\left(x_{0}, z_{0} ; \varphi_{0}\right)$ é a equação (2.13) resolvida por $\varphi$. Tal equação diferencial será a equação iconal da ondaimagem.

Derivando a equação (2.13) em relação a $z$, chegamos a

$$
1=\frac{-1}{2 z}\left[\Phi_{0} \Phi_{z} \frac{\left(x-x_{0}\right)^{2}}{\Phi-\Phi_{0}}-\Phi_{0} \Phi \frac{\left(x-x_{0}\right)^{2}}{\left(\Phi-\Phi_{0}\right)^{2}} \Phi_{z}\right]=\frac{\Phi_{z}}{2 z} \frac{\Phi_{0}^{2}\left(x-x_{0}\right)^{2}}{\left(\Phi-\Phi_{0}\right)^{2}} .
$$

A partir da igualdade acima, obtemos

$$
\frac{\Phi_{0}^{2}\left(x-x_{0}\right)^{2}}{\left(\Phi-\Phi_{0}\right)^{2}}=\frac{2 z}{\Phi_{z}} .
$$

Diferenciação da equação (2.13) em relação a $x$, resulta em

$$
0=\frac{-1}{2 z}\left[-\Phi_{x} \frac{\Phi_{0}^{2}\left(x-x_{0}\right)^{2}}{\left(\Phi-\Phi_{0}\right)^{2}}+\Phi \Phi_{0} \frac{2\left(x-x_{0}\right)}{\Phi-\Phi_{0}}\right]=\frac{-1}{2 z}\left[\frac{\Phi_{x}}{\Phi_{z}} 2 z+2 \Phi \frac{\Phi_{0}\left(x-x_{0}\right)}{\Phi-\Phi_{0}}\right]
$$

e, portanto,

$$
\frac{\Phi_{x}}{\Phi_{z}}=\frac{\Phi}{z}\left[\frac{\Phi_{0}\left(x-x_{0}\right)}{\Phi-\Phi_{0}}\right] .
$$

Elevando esta expressão ao quadrado e substituindo nela a equação (2.14), obtemos

$$
\frac{\Phi_{x}^{2}}{\Phi_{z}^{2}}=\frac{\Phi^{2}}{z^{2}} \frac{2 z}{\Phi_{z}} \Rightarrow \frac{\Phi_{x}^{2}}{\Phi_{z}^{2}}=\frac{2 \Phi^{2}}{z \Phi_{z}} .
$$

Simplificando a equação acima, chegamos à equação iconal da onda-imagem

$$
\Phi_{x}^{2}-\frac{2 \Phi^{2}}{z} \Phi_{z}=0
$$


Equação da onda-imagem: Agora queremos encontrar uma equação diferencial parcial tal que a equação acima seja a equação iconal associada. Para isso utilizamos a candidata correspondente à teoria dos raios

$$
p(x, z, \varphi)=p_{0}(x, z) f[\varphi-\Phi(x, z)] .
$$

Derivando a candidata (2.16) duas vezes com respeito a $x$, temos

$$
\begin{aligned}
p_{x x}= & p_{0 x x} f(\varphi-\Phi)-2 p_{0 x} f^{\prime}(\varphi-\Phi) \Phi_{x} \\
& +p_{0} f^{\prime \prime}(\varphi-\Phi)\left(\Phi_{x}\right)^{2}-p_{0} f^{\prime}(\varphi-\Phi) \Phi_{x x} .
\end{aligned}
$$

Correspondentemente, a derivada mista com respeito a $z$ e $\varphi$ é

$$
p_{z \varphi}=p_{0 z} f^{\prime}(\varphi-\Phi)-p_{0} f^{\prime \prime}(\varphi-\Phi) \Phi_{z} .
$$

Aplicando a metodologia inversa das equações (2.1) até (2.4), e observando os termos que multiplicam $f^{\prime \prime}$, devemos ter

$$
p_{x x}+\frac{2 \varphi^{2}}{z} p_{z \varphi}=0
$$

para que assim a substituição da candidata (2.16) possa reproduzir a equação iconal (2.15). Note que a equação acima é a mais simples que tem a propriedade desejada. Termos adicionais envolvendo as primeiras derivadas de $p$ com respeito a $x, z$, ou $\varphi$ não alterariam a equação iconal associada e, portanto, também não o comportamento cinemático da solução. Como estamos interessados neste momento somente no comportamento cinemático correto da solução, podemos então optar por esta forma mais simples. Chamamos a equação (2.19) de equação da onda-imagem para remigração na profundidade em meios elipticamente anisotrópicos.

\section{Conclusão}

A variação da imagem de um refletor sísmico sob mudança do modelo de velocidade pode ser entendida de maneira análoga à propagação de uma onda $[2,4]$.

Neste trabalho, deduzimos uma equação diferencial parcial de segunda ordem que funciona como equação da onda-imagem para remigração em profundidade em meios elipticamente anisotrópicos sob a variação do parâmetro de elipticidade. Estudamos a cinemática da onda-imagem nestes meios para deduzir a respectiva equação iconal. Invertendo a metodologia da teoria dos raios, esta nos fornece a desejada equação onda-imagem em tal meio.

A propagação de uma imagem em função do parâmetro de elipticidade é muito desejável na sísmica, uma vez que a velocidade de migração correta geralmente não é conhecida e migrações com vários valores dos parâmetros se tornam necessárias. $\mathrm{O}$ objetivo da equação da onda-imagem deduzida é possibilitar a determinação de um conjunto de imagens do subsolo correspondentes a diferentes valores do parâmetro utilizando a equação da onda-imagem ao invés de múltiplas migrações, com o intuito de escolher entre elas a geologicamente mais fidedigna. 
Uma aplicação importante desta equação surge quando o modelo usado para a migração inicial for um meio isotrópico, i.e., quando nas condições iniciais temse $\varphi_{0}=1$. É nesta situação que a presente estratégia da remigração por ondaimagem se torna especialmente interessante, uma vez que a migração em meios isotrópicos é um processo muito bem conhecido. Desta forma, poderá-se aplicar a um processo elaborado de migração isotrópica, seguido de uma remigração por onda-imagem para transformar a imagem migrada obtida em um meio isotrópico em uma imagem referente a um meio anisotrópico. É importante observar, neste contexto, que a equação (2.19) não depende explicitamente da velocidade vertical, assim indicando seu potencial até em meios não-homogêneos.

\title{
Agradecimentos
}

Os autores agradecem o apoio financeiro da CAPES, CNPq e aos patrocinadores do consórcio Wave Inversion Technology (WIT).

\begin{abstract}
The image of a seismic reflector changes position in depth when remigrated under variation of the velocity model. This "propagation" can be described by means of a second-order partial differential equation similar to the acoustic wave equation. In this work, we take the derivation of this so-called image-wave equation to elliptically anisotropic media, utilizing the geometric properties of the seismic remigration problem. Like in the isotropic case, the derivation uses a methodology that amounts to a reverse application of standard ray theory. The aim is the construction of subsurface images that correspond to different degrees of anisotropy by means of an image propagation instead of multiple migrations.
\end{abstract}

\section{Referências}

[1] V. Červený, "Seismic Ray Theory", Cambridge University Press, Cambridge, 2001.

[2] S.B. Fomel, Method of velocity continuation in the problem of seismic time migration, Russian Geology and Geophysics, 35, No. 5 (1994), 100-111.

[3] K. Helbig, Elliptical anisotropy - its significance and meaning, Geophysics, 48 (1983), 825-832.

[4] P. Hubral, M. Tygel e J. Schleicher, Seismic Image Waves, Geophys. J. Internat., 125 (1996), 431-442.

[5] P. Hubral, J. Schleicher e M. Tygel, A unified aproach to seismic reflection imaging - Part I: Basic Concepts, Geophysics, 61 (1996), 742-758.

[6] M.S. Jaya, "Imaging Reflection Seismic Data Using the Method of Velocity Continuation", Tese de Doutorado, Universität Karlsruhe, Karlsruhe, Alemanha, 1997.

[7] C. Vanelle, A tutorial on elliptical anisotropy, Annual WIT Report., 6 (2002), $267-275$ 